\title{
Association between genetic obesity susceptibility and mother-reported eating behavior in children up to 5 years
}

Blandine de Lauzon-Guillain, $\mathrm{PhD}^{1,2,8}$, Yves Akoli Koudou, $\mathrm{MSc}^{1}$, Jérémie Botton, $\mathrm{PhD}^{1,3}$, Anne Forhan, $\mathrm{MSc}^{1,2}$, Sophie Carles, $\mathrm{PhD}^{1}$, Véronique Pelloux, $\mathrm{PhD}^{4,5,6}$, Karine Clément, $\mathrm{PhD}^{4,5,6}$, Ken K. Ong, FRCPCH${ }^{7}$, Marie Aline Charles, MD, $\mathrm{PhD}^{1,2}$, Barbara Heude, $\mathrm{PhD}^{1,2}$; on behalf of the EDEN Mother-Child Cohort Study Group*

* Members of the EDEN Mother-Child Cohort Study Group: I. Annesi-Maesano, JY. Bernard, J. Botton, M.A. Charles, P. Dargent-Molina, B. de Lauzon-Guillain, P. Ducimetière, M. de Agostini, B. Foliguet, A. Forhan, X. Fritel, A. Germa, V. Goua, R. Hankard, B. Heude, M. Kaminski, B. Larroque $\dagger$, N. Lelong, J. Lepeule, G. Magnin, L. Marchand, C. Nabet, F. Pierre, R. Slama, M.J. Saurel-Cubizolles, M. Schweitzer, O. Thiebaugeorges.

\section{Affiliations}

${ }^{1}$ INSERM, UMR1153 Epidemiology and Biostatistics Sorbonne Paris Cité Center, Early ORigin of the Child's Health and Development Team (ORCHAD), Paris, France

${ }^{2}$ Paris Descartes University, France

${ }^{3}$ Univ. Paris-Sud, Université Paris-Saclay, Faculty of Pharmacy, F-92296, ChâtenayMalabry, France;

${ }^{4}$ Institute of Cardiometabolism and Nutrition, ICAN, F-75013, Paris, France;

${ }^{5}$ INSERM, UMRS 1166, Nutriomic team 6, Paris, F-75013 France;

${ }^{6}$ Sorbonne Universités, UPMC Université Paris 06, UMRS1166, Paris, F-75013 France;

${ }^{7}$ Medical Research Council Epidemiology Unit \& Department of Paediatrics, University of Cambridge, Addenbrooke's Hospital, Cambridge, England

${ }^{8}$ INRA, U1125 Epidemiology and Biostatistics Sorbonne Paris Cité Center (CRESS), Early ORigin of the Child's Health and Development Team (ORCHAD), Paris, F-75014 France. 


\section{PubMed indexing.}

de Lauzon-Guillain, Koudou, Botton, Forhan, Carles, Pelloux, Clément, Ong, Charles, Heude; on behalf of the EDEN Mother-Child Cohort Study Group

\section{Running head}

genetic obesity risk and child's eating behavior

\section{Keywords}

obesity, genetic, eating behaviour, growth, birth cohort, childhood

\section{Corresponding author}

de Lauzon-Guillain Blandine

INSERM CRESS - Eq6 ORCHAD

16 av. Paul Vaillant Couturier, 94807 Villejuif Cedex, FRANCE

Tel: +33145595019; Fax: +33147269454; E-mail: blandine.delauzon@inserm.fr

\section{Abbreviations}

BMI-GRS: combined obesity risk-allele score

SNP: single-nucleotide polymorphisms 


\section{$1 \quad \underline{\text { Abstract }}$}

2 Background. Many genetic polymorphisms identified by genome wide association studies

3 for adult BMI have been suggested to regulate food intake.

4 Objective. The objective was to study the associations between a genetic obesity risk score,

5 appetitive traits and growth of children up to age 5 years, with a longitudinal design.

6 Methods. In 1,142 children from the EDEN birth cohort, a combined obesity risk-allele score

7 (BMI-GRS) was related to appetitive traits (energy intake up to 12 months, a single item on

8 appetite from 4 months to 3 years, a validated appetite score at 5 years) using Poisson

9 regressions with robust standard errors. The potential mediation of appetitive traits on the

10 association between BMI-GRS and growth was assessed by the Sobel test.

11 Results. Children with a high BMI-GRS were more likely to have high energy intake at 1

12 year and high appetite at 2 and 5 years. High energy intake in infancy and high appetite from

131 year were related to higher subsequent BMI. High 2-y appetite seemed to partially mediate

14 the associations between BMI-GRS and BMI from 2 to 5 years (all $p<=0.05$ ).

15 Conclusions. Genetic susceptibility to childhood obesity seems to be partially explained by

16 appetitive traits in infancy, followed by an early childhood rise in BMI. 


\section{Introduction}

Genome-wide-association studies (GWAs) have identified several genes associated with an increased risk of obesity $(1,2)$. Time has now come to take advantage of these major findings in order to better understand the mechanisms of lifecourse obesity development from infancy to adulthood. Many birth cohorts have shown that scores of genetic susceptibility to obesity are associated with early growth, as early as 2 years in some of them, but not with foetal growth $(3,4,5,6)$.

A great part of the genes implicated by GWAs, and especially those from monogenic studies on severe childhood or early onset obesity, are purportedly involved in the central regulation of food intake (7). Furthermore, the genetic component of eating behavior has clearly been established through heritability studies, in adults (8) and also in children (9, 10). Eating behavior even in infancy may influence later adiposity development (11), which raises the question whether the association between the genetic score and adiposity is mediated by eating behaviour in childhood. Cross-sectional studies, conducted among 8-11 years children, have already shown that increased BMI of children homozygous for the at-risk allele of a FTO SNP may be partially mediated by appetitive traits $(12,13)$. Similarly, a cross-sectional analysis of the Twins Early Development Study highlighted that satiety responsiveness partially mediated the association between a polygenic risk score and adiposity in children aged 8-11 years(14). However, such a cross-sectional design is insufficient to establish a causal pathway. More recently, this causal pathway has been tested longitudinally within a Norwegian cohort following children from the age of 4 years to the age of 8 years (15). In that study, higher genetic risk for obesity was associated with appetitive traits at 6 years, but these appetitive traits were not related to BMI gain up to 8 years. As a previous study has shown that appetitive traits assessed at age 3 months predicted weight at 9 months, it would be important to test the potential mediating effect of infant or toddler's appetitive traits on 
subsequent growth. A prospective design would also offer the opportunity to highlight a potential window of opportunity for this mediating effect.

In this context, we aimed at investigating within the French EDEN mother-child cohort, how genetic predisposition to obesity may influence eating behavior very early life and whether this relationship might explain the accelerated growth in children with higher genetic risk for obesity. In the present study, we focused on three aspects of child's eating behavior that could be assessed longitudinally, from 4 months to 5 years: energy intake and appetite.

\section{Material and methods}

\section{Study population}

The EDEN mother-child study is a prospective cohort whose aim is to assess pre- and postnatal determinants of child growth, development and health. It has been described in more detail elsewhere (16). In brief, 2002 pregnant women were recruited from 2003 to 2006 in two French university hospitals, before 24 weeks of amenorrhea. Exclusion criteria were multiple pregnancies, known diabetes prior to pregnancy, illiteracy, plan to move outside the region within the next three years. The study was approved by the Ethics Committee of the University Hospital of Kremlin-Bicêtre on December 12, 2002 and data files were declared to the National Committee for Processed Data and Freedom. Written consent was obtained from both parents.

The primary endpoints of the cohort were pre and post-natal growth, blood pressure, allergies, infectious diseases, mental health and cognitive development. Dietary behavior was initially collected as one of the main exposure factors in the cohort and was considered as a potential mediating factor in the present study. 
Data collected during pregnancy and at birth, include sociodemographic variables, maternal smoking, gestational diabetes, parental anthropometric measurements, and newborn characteristics (sex, gestational age, birthweight) (17). At four, eight and twelve months after birth, mothers completed mailed questionnaires that provided detailed information on the feeding method (exclusive breast-feeding, exclusive formula-feeding or mixed-diet) between birth and four months, then at eight and twelve months as well as the date of breastfeeding cessation. Breastfeeding duration was derived from these data (18). Clinical exams were also conducted at birth, 1 year, 3 years and 5 years and were dedicated to collection of biological sample, cognitive assessments by psychologists and measurement of anthropometric and clinical parameters.

\section{Genotyping and BMI Genetic Risk Score (BMI-GRS) generation}

DNA was extracted from cord blood samples collected at birth. Genotypes at 16 singlenucleotide polymorphisms (SNPs) were measured at the Medical Research Council Epidemiology Unit, Cambridge (iPLEX platform; Sequenom), as previously described (19). Among the 32 loci identified by Speliotes et al. as having genome-wide significant associations with BMI in adults (2), we considered in the present study the 16 SNPs also showing associations with childhood BMI either in that original report (2) or in subsequent data (4); they lie in or near NRXN3 (rs10146997), SLC39A8 (rs13107325), TNNI3K (rs 1514175), PTBP2 (rs1555543), MC4R (rs17782313), FLJ35779 (rs2112347), NEGR1 (rs2568958), RPL27A (rs4929949), TMEM18 (rs6548238), RBJ/POMC (rs713586), CADM2 (rs7640855), TRA2B/ETV5 (rs7647305), BDNF (rs925946), TFAP2B (rs987237), FTO (rs9941349), and ZNF608 (rs4836133). The summary of genotyped SNPs and allele frequencies in presented in supplementary table S1. All variants passed genotyping quality control criteria (call rate, >95\%; Hardy-Weinberg equilibrium, $\mathrm{P}>.01$ ). 
Combined obesity risk-allele scores, indicating genetic susceptibility to obesity, were calculated for each participant as the sum of risk alleles ( 0,1 or 2 at each locus) associated with higher BMI across the 16 SNP loci. The score ranged from 0 to 32 . To minimize dropout due to missing genotype data, infants with missing genotype data at $4(25 \%)$ or fewer loci were imputed with the mean number of susceptibility alleles in their cohort for each locus. In sensitivity analyses, we also computed a weighted BMI-GRS, where each risk allele was multiplied by the 'European only sex combined' effect estimate for the BMI-increasing allele reported by Locke and colleagues (1).

\section{Child's eating behavior}

Maternal perception of infant's appetite was assessed, at ages 4, 8 and 12 months, by one question, translated as follow: "Usually, you would say that your baby: 1/ is always hungry or demanding to feed 2 / demands to feed the same as other babies of the same age $3 /$ needs to be stimulated to eat", at ages 2 and 3 years, by one question: "Usually, you would say that your child: 1 / is always hungry or asking to eat 2 / has a normal appetite compared with other children of the same age 3 / is not often hungry", and at 5 years by the Low Appetite scale from the Children's Eating Difficulties Questionnaire (20). From 4 months to 4 years, a high appetite was defined as the category of children "always hungry or claiming to eat" and at 5 years as a Low Appetite score below the $10^{\text {th }}$ percentile.

Infant diet was assessed by food records on three non-consecutive days (two weekdays and one weekend day), when the infant was not sick, at 4, 8 and 12 months. Data were checked and computerized a posteriori by a dietician. Nutrient intake was then calculated based on two food composition databases, one specific to ready-prepared baby foods from the French baby foods industry group (SFAE 2005) and one for common foods from the French Observatory of Food Nutritional Quality (CIQUAL 2006) (21). Total nutrient intake was calculated only among infants who were not breastfed during the three days of the dietary records, given that 
114 the amount of breast milk intake could not be measured in breastfed infants. At each age, high

115 energy intake was defined as the highest quintile of energy intake. Unfortunately, energy

116 intake was not available in 2-5-year-old children, as diet was collected from a 24-item food

117 frequency questionnaire from 2 years instead of 3-d dietary records.

\section{Child's growth}

119 At each clinical examination, child's weight and height were measured. In between, weight

120 and height data were collected from self-administered questionnaires and from measurements

121 noted in the child's health booklet by health professionals. Children had on average 22 weight

122 measurements (interquartile range 16-26) from birth to 5-6 years. Individual growth curves

123 were obtained for weight and height using the Jenss-Bayley growth curve model (22). This

124 model allows us to predict weight, length/height and then calculate BMI at any given age

125 (22). We used these model-based values of weight, length/height and BMI at 1, 2, 3, 4 and 5

126 y. At each age, the WHO growth standards were used for the calculation of weight-for-age,

127 length/height-for-age and BMI-for-age z-scores. The WHO Anthro SAS macro (WHO

128 Anthro, SAS Macro, Geneva: World Health Organization;

129 http://www.who.int/childgrowth/software/en/) was used for this calculation.

\section{Sample selection}

131 Of the 2002 women who were recruited, 76 women were excluded because they left the study

132 before or at the time of delivery, 24 because of miscarriages, intra-uterine death, or

133 discontinuation of pregnancy for medical reasons, and 9 because they delivered outside the

134 study hospitals. Data on birthweight were available for 1899 newborns. Among them,

135 genotyping data were available for 1324 children. Children without any data on eating

136 behavior from 4 months to 5 years $(n=123)$ as well as those with missing data on age, sex,

137 growth, breastfeeding duration or maternal smoking $(n=59)$ were excluded from the analyses.

138 Among the 1899 newborns with data on birth weight, the 1142 participants included in our 
139 analyses were quite similar to those excluded concerning gender $(\mathrm{p}=0.9)$ and maternal pre-

140 gestational BMI ( $\mathrm{p}=0.12)$ but had older mothers (29.7 vs 29.1 years at delivery, $\mathrm{p}=0.007)$ and

141 were born with higher gestational age (39.4 vs. 39.0 weeks of amenorrhea, p<0.0001) and

142 higher birth weight (3324 g vs. $3210 \mathrm{~g}, \mathrm{p}<0.0001)$.

\section{Statistical analyses}

144 Student $t$-tests, $\chi^{2}$-tests and 1-way ANOVA were used to test differences between included 145 and excluded populations.

146 Associations between the obesity risk-allele score (BMI-GRS) and child's eating behavior

147 were tested by Poisson regression models with robust standard errors, adjusted for recruitment

148 center, infant's gender, and age at eating behavior assessment. Additional analyses were

149 conducted to adjust further for breastfeeding duration and maternal smoking during

150 pregnancy.

151 Associations between infant/child eating behavior and predicted child's weight-for-age,

152 length/height-for age or BMI-for-age WHO z-scores were tested by linear regression models

153 adjusted for recruitment center, infant's sex, age at eating behavior assessment. Additional

154 analyses were conducted to adjust further for breastfeeding duration, maternal smoking during

155 pregnancy and parental height.

156 Associations between BMI-GRS and predicted child's weight, length/height or BMI z-scores

157 were tested by linear regression models adjusted for recruitment center and infant's sex.

158 If an eating behaviour was associated with both the independent variable (BMI-GRS, path a,

159 Supplementary Figure S1) and the dependent variable (WHO z-score, path b), we tested for

160 the presence of mediation. Mediation is said to occur when a third variable lies on the causal

161 pathway between an exposure (in this case, the BMI-GRS) and an outcome (in this case,

162 WHO z-scores). To test for mediation, the linear regression of genetic risk score on each

163 WHO z-score was adjusted for the considered eating behaviour. The presence of mediation 
164 was established using the Sobel test $(23,24)$ and quantified by the mediation ratio $\left(\left(\beta-\beta^{\prime}\right) / \beta\right)$,

165 where $\beta$ is the initial coefficient for BMI from the model WHO z-score $\sim B M I-G R S$ (path c)

166 and $\beta^{\prime}$ is the coefficient for WHO z-score after the model is additionally adjusted for the

167 eating behaviour (path c'). As high score on appetitive traits were considered as binary

168 variables, we used the method adapted by Jasti et al. to calculate the Sobel test (25).

169 We conducted sensitivity analyses using a weighted genetic risk score for BMI (WtBMI-

170 GRS) instead ot the crude genetic risk score. To calculate this weighted score, at each locus,

171 the number of BMI increasing variants was multiplied by the effect estimate for the BMI-

172 increasing variant from Speliotes et al (2).

173 All analyses were carried out using SAS V9.3 (SAS, Cary, NC).

\section{$174 \underline{\text { Results }}$}

175 Among the 1142 mothers-child pairs with both genotyping data and eating behavior

176 assessment, mothers were aged on average $29.7(\mathrm{SD}=4.8)$ years, had a pre-pregnancy BMI of

$17723.1(\mathrm{SD}=4.3) \mathrm{kg} / \mathrm{m}^{2}$ and breastfed for $3.3(\mathrm{SD}=3.7)$ months. Children carried on average 13.7

$178(\mathrm{SD}=2.5)$ obesity risk alleles, with a range of 5 to 22 obesity risk alleles. At 5 years, $6.6 \%$ of

179 children were considered overweight or obese using IOTF definition (26). Children's BMI

180 and appetitive traits are described in Supplementary table S2.

\section{BMI-GRS related to eating behaviours}

182 BMI-GRS was not significantly related to high energy intake at 4 mo and 8 mo but was

183 positively related to energy intake at 1 year (in infants who were not breastfed at time of

184 energy intake assessment) (Figure 1). Among all children, BMI-GRS was positively related

185 to maternal perception of high appetite between 8 months and 5 years, although not

186 significant in the first year or at 3 years. Further adjustment for breastfeeding duration and

187 maternal smoking during pregnancy did not substantially modify the associations (data not 
188 shown). In sensitivity analyses, when considering the weighted BMI-GRS, results were

189 similar (data not shown).

\section{Child's eating behavior and growth parameters}

191 Among children not breastfed during the 3-d dietary records, high energy intakes at 4 and 8

192 months were positively related to weight-for-age, length/ height-for-age and BMI-for-age z-

193 scores (Table 1) from 1 to 5 years. High energy intake at 12 months was positively related to

194 weight-for-age z-score from 3 to 5 years but was not related to length/ height-for-age or BMI-

195 for-age z-scores.

196 High appetite before 1 year was not clearly related to subsequent WHO's z-scores. High

197 appetite was not related to length/height-for-age z-score, whatever the age considered. High

198 appetite at 1 year was positively related to weight-for-age or BMI-for-age z-scores up to 5

199 years but the strength of the association decreased with age and was no more significant for

200 BMI-for-age at 5 years. High appetite at 2 or 3 years was positively related to child's

201 subsequent BMI-for-age z-score and, to a lesser extent, weight-for-age z-score. These

202 associations were mitigated by further adjustment for breastfeeding duration, maternal

203 smoking during pregnancy and parental height, the associations but remained significant,

204 except the association between high energy intake at 12 months and weight-for-age z-score

205 from 3 to 5 years (supplementary table S3)

\section{Mediation analysis}

207 The association between BMI-GRS and child's size increased with age and became

208 significant from 3 years onwards for child's weight (Figure 2A) and 4 years onwards for

209 child's BMI (Figure 2B). High appetite at 2 years was the only eating behavior related to

210 both BMI-GRS and BMI, and therefore considered as a potential mediator in the association

211 between BMI-GRS and BMI. These associations were attenuated after adjustment for high

212 appetite at 2 years (Figure 2A and 2B). The Sobel test for mediation was significant but the 
213 mediation was only partial with a mediation ratio decreasing with time from $47 \%$ for 2 -y BMI

214 z-score $(\mathrm{p}=0.03)$ and $35 \%$ for 3 -y BMI z-score $(\mathrm{p}=0.03)$ to $28 \%$ for 4-y BMI $\mathrm{z}$-score $(\mathrm{p}=0.05)$

215 and $24 \%$ for 5-y BMI ( $\mathrm{p}=0.05$ ). Similar results were found with the weighted BMI-GRS (data

216 not shown).

217 Discussion

218 In our study, the score of genetic predisposition to develop obesity was association with a

219 higher risk of high energy intake at 1 year, and high parental-perceived appetite at 2 and 5

220 years. The associations between the BMI-GRS and BMI-for-age or, to a lesser extent, weight-

221 for-age $\mathrm{z}$-scores were attenuated by further adjustment for high appetite at 2 years, whereas

222 BMI-GRS was not related to length/height-for-age z-score up to 5 years. Finally, among

223 infants not breastfed during the food records, energy intakes at 4 and 8 months were

224 positively related to child's WHO z-scores until 5 years, but were not related to the BMI-

225 GRS.

227 The association between genetic susceptibility to obesity and appetitive traits has been

228 previously examined in childhood but not so early in infancy. Within 8-11 years olds twins of

229 the TEDS study, Llewellyn et al. (14) showed that a BMI-GRS (based on 28 SNPs) was

230 related to both BMI and satiety sensitivity, and that the latter mediated the association with

231 BMI. However, in a study conducted by Steinsbeckk et al. within Norwegian children aged

232 from 4 to 6 years at inclusion (15), the BMI-GRS (based on 32 SNPs) was related to BMI

233 from 4 to 8 years, to BMI change from 4 to 6 years and from 6 to 8 years, but was associated

234 only with slowness in eating (negative association) at 6 years and not with other eating

235 behaviours (i.e. food responsiveness, emotional overeating, enjoyment of food or satiety

236 responsiveness). In that study, the association between the BMI-GRS and BMI was not

237 mediated by any appetitive trait assessed at age 6 years. 
238 If evidence of a mediating effect of appetitive traits on the association between the BMI-GRS

239 and BMI remains scarce, many studies examined the influence of appetitive traits on BMI.

240 The associations shown in EDEN are in agreement with previous studies in spite of the

241 heterogeneity of ages and methods of investigation. In the GUSTO study (27), food

242 responsiveness at 3 months was related to higher BMI up to 15 months, whereas appetitive

243 traits assessed at 12 months were not related to BMI z-score in toddlers. Moreover, satiety

244 responsiveness in early childhood was found related to lower subsequent BMI $(28,29)$.

245 Similar results were found in the Gemini study (11), as all appetitive traits measured at 3

246 months of age were related to later weight, with stronger association for general appetite and

247 satiety responsiveness. In that study, the possibility of reverse causality was suggested by the

248 observation that weight at 9 months was also related to appetitive traits in toddlers, with

249 stronger association for food responsiveness, satiety responsiveness and general appetite.

250 Despite the positive associations between infant appetite and subsequent weight gain and also

251 between infant size and subsequent, the prospective influence of appetite on weight appeared

252 to be stronger than the reverse.

253 In our study, energy intake in the first months of life was related to growth throughout early

254 childhood. High energy intake could probably be considered as an indicator of rapid growth

255 during this period and rapid growth in infancy is related to higher risk of overweight later in

256 childhood in this and other cohorts (30). The influence of high energy intake on child's 5-y

257 BMI is similar to the influence of high genetic predisposition to obesity (data not shown).

258 However, notably energy intake in infancy was not related to the BMI-GRS, and might be

259 more explained by prenatal or early postnatal exposures. We have to acknowledge that energy

260 intake was assessed only among infants who were not breastfed during the three days of the

261 dietary records, given that the amount of breast milk intake could not be measured in 
262 breastfed infants. Moreover, from 2 years onwards, the dietary data collection was

263 unfortunately not designed to derive energy intake.

265 The main strength of this study is that eating behaviour and BMI were assessed repetitively 266 and prospectively from very early in infancy (as early as birth for BMI and four months for

267 eating behaviour), which permitted us to describe in detail the longitudinal patterns of 268 associations between the BMI-GRS, eating behaviour and BMI. However, appetite was 269 assessed only by one item, whereas other assessments, maybe based on more complex scales, 270 would have allowed us to check for consistency for this trait. Moreover, we have to 271 acknowledge that eating behaviour up to 3 years was not assessed by a validated tool up to 3 272 years as such tools were not developed when the EDEN study was designed. A validated tool 273 became available and was introduced only at the 5-y follow-up. Concerning the item on 274 appetite, a similar item was used by previous studies $(10,11)$ and associations with this item 275 were similar to those with other appetitive traits, if not stronger. Moreover, in the EDEN 276 study, we found similar associations at 5 years, when appetite was assessed by a validated 277 scale (20). As appetitive traits were reported by parental questionnaires and not assessed by a 278 laboratory tool, this measurement could be influenced by social desirability. Finally, because 279 of the originality and the uniqueness of the collected data, our results yet rely on data from 280 only one cohort. Further studies conducted in other birth cohorts would be necessary to 281 confirm and demonstrate the generalizability of these findings. Ideally, these studies would be 282 designed with repeated use of validated tools throughout early childhood to assess eating 283 behaviour or energy intake and conducted in populations with higher prevalence of 284 overweight. As previous studies have shown that these BMI variants could to be relevant also 285 to other non-Caucasian populations $(31,32)$, it would be interesting to conduct similar 286 analyses in non-Caucasian populations. 


\section{Conclusion}

289 The study provides further support from a prospective study for a partial mediation by

290 appetitive traits on the relationship between genetic susceptibility and BMI. In particular, in

291 the EDEN birth cohort, genetic susceptibility to childhood and adult obesity seems to play a

292 very precocious role by influencing appetitive traits in infants and preschool children and

293 subsequently promote faster growth. Despite the more typically recognised concerns

294 regarding infant feeding difficulties, many parents might benefit from counselling and support

295 to manage high appetitive traits in their infants and young children to prevent later obesity

296 risk. 


\section{$\underline{\text { Sources of support }}$}

299 The EDEN study is supported by: Fondation pour la Recherche Médicale (FRM), French

300 Ministry of Research: Federative Research Institutes and Cohort Program, INSERM Human

301 Nutrition National Research Program, and Diabetes National Research Program (through a 302 collaboration with the French Association of Diabetic Patients (AFD)), French Ministry of Health, French Agency for Environment Security (AFSSET), French National Institute for Population Health Surveillance (InVS), Paris-Sud University, French National Institute for Health Education (INPES), Nestlé, Mutuelle Générale de l'Education Nationale (MGEN), French speaking association for the study of diabetes and metabolism (ALFEDIAM), National Agency for Research (ANR non thematic program), National Institute for Research in Public health (IRESP: TGIR 2008 cohort in health program). The genotyping was funded by a Collaborative Research Grant from the European Society for Paediatric Endocrinology. KKO is supported by the Medical Research Council (Unit program: MC_UU_12015/2).

\section{Acknowledgment section}

312 None of the authors have any financial relationships or conflict of interest to disclose.

BLG and $\mathrm{BH}$ designed the research and wrote the manuscript. BLG, YAK, JB, AF and SC analysed the data. $\mathrm{BH}$ and MAC oversaw the EDEN study. BH, KC, VP, KKO and MAC were responsible for data collection and genotyping in EDEN. All authors reviewed drafts,

317 provided critical feedback, approved the final manuscript and were responsible for the final 318 content of the paper. BH had full access to all the data in the study and takes responsibility for the integrity of the data and the accuracy of the data analysis. 


\section{$\underline{\text { References }}$}

1. Locke AE, Kahali B, Berndt SI, et al. Genetic studies of body mass index yield new insights for obesity biology. Nature 2015; 518, 197-206.

2. Speliotes EK, Willer CJ, Berndt SI, et al. Association analyses of 249,796 individuals reveal 18 new loci associated with body mass index. Nat Genet 2010; 42, 937-48.

3. Belsky DW, Moffitt TE, Houts R, et al. Polygenic risk, rapid childhood growth, and the development of obesity: evidence from a 4-decade longitudinal study. Arch Pediatr Adolesc Med 2012; 166, 515-21.

4. Elks CE, Heude B, de Zegher F, et al. Associations between genetic obesity susceptibility and early postnatal fat and lean mass: an individual participant metaanalysis. JAMA Pediatr 2014; 168, 1122-30.

5. Elks CE, Loos RJ, Hardy R, et al. Adult obesity susceptibility variants are associated with greater childhood weight gain and a faster tempo of growth: the 1946 British Birth Cohort Study. Am J Clin Nutr 2012; 95, 1150-6.

6. Elks CE, Loos RJ, Sharp SJ, et al. Genetic markers of adult obesity risk are associated with greater early infancy weight gain and growth. PLoS Med 2010; 7, e1000284.

7. Day FR and Loos RJ. Developments in obesity genetics in the era of genome-wide association studies. J Nutrigenet Nutrigenomics 2011; 4, 222-38.

8. $\quad$ Elder SJ, Neale MC, Fuss PJ, et al. Genetic and Environmental Influences on Eating Behavior - A Study of Twin Pairs Reared Apart or Reared Together. Open Nutr J 2012; 6, 59-70.

9. Llewellyn $\mathrm{CH}$, van Jaarsveld $\mathrm{CH}$, Boniface D, Carnell S and Wardle J. Eating rate is a heritable phenotype related to weight in children. Am J Clin Nutr 2008; 88, 1560-6.

10. Llewellyn $\mathrm{CH}$, van Jaarsveld $\mathrm{CH}$, Plomin $\mathrm{R}$, Fisher $\mathrm{A}$ and Wardle J. Inherited behavioral susceptibility to adiposity in infancy: a multivariate genetic analysis of appetite and weight in the Gemini birth cohort. Am J Clin Nutr 2012; 95, 633-9.

11. van Jaarsveld $\mathrm{CH}$, Llewellyn $\mathrm{CH}$, Johnson $\mathrm{L}$ and Wardle J. Prospective associations between appetitive traits and weight gain in infancy. Am J Clin Nutr 2011; 94, 1562-7.

12. Emond JA, Tovar A, Li Z, Lansigan RK and Gilbert-Diamond D. FTO genotype and weight status among preadolescents: Assessing the mediating effects of obesogenic appetitive traits. Appetite 2017; 117, 321-329.

13. Wardle J, Carnell S, Haworth CM, Farooqi IS, O'Rahilly S and Plomin R. Obesity associated genetic variation in FTO is associated with diminished satiety. J Clin Endocrinol Metab 2008; 93, 3640-3.

14. Llewellyn $\mathrm{CH}$, Trzaskowski M, van Jaarsveld $\mathrm{CH}$, Plomin R and Wardle J. Satiety mechanisms in genetic risk of obesity. JAMA Pediatr 2014; 168, 338-44.

15. Steinsbekk S, Belsky D, Guzey IC, Wardle J and Wichstrom L. Polygenic Risk, Appetite Traits, and Weight Gain in Middle Childhood: A Longitudinal Study. JAMA Pediatr 2016; 170, e154472.

16. Heude B, Forhan A, Slama R, et al. Cohort Profile: The EDEN mother-child cohort on the prenatal and early postnatal determinants of child health and development. Int J Epidemiol 2016; 45, 353-63.

17. Regnault N, Botton J, Forhan A, et al. Determinants of early ponderal and statural growth in full-term infants in the EDEN mother-child cohort study. Am J Clin Nutr 2010; 92, 594-602. 
18. Betoko A, Charles MA, Hankard R, et al. Infant feeding patterns over the first year of life: influence of family characteristics. Eur J Clin Nutr 2013; 67, 631-637.

19. Li S, Zhao JH, Luan J, et al. Cumulative effects and predictive value of common obesity-susceptibility variants identified by genome-wide association studies. Am J Clin Nutr 2010; 91, 184-90.

20. Rigal N, Chabanet C, Issanchou S and Monnery-Patris S. Links between maternal feeding practices and children's eating difficulties. Validation of French tools. Appetite 2012; 58, 629-37.

21. ANSES. French food composition table [internet] 2015, France: French Agency for Food, Environmental and Occupational Health \& Safety (ANSES). 2015.

22. Botton J, Scherdel P, Regnault N, Heude B, Charles MA and Eden Mother-Child Cohort Study Group. Postnatal weight and height growth modeling and prediction of body mass index as a function of time for the study of growth determinants. Ann Nutr Metab 2014; 65, 156-66.

23. Mackinnon DP, Warsi G and Dwyer JH. A Simulation Study of Mediated Effect Measures. Multivariate Behav Res 1995; 30, 41.

24. Sobel ME. Asymptotic intervals for indirect effect in structural equations models. In Sociological methodology, ed. Leinhart S, 1982, pp 290-312 San Francisco: JosseyBass.

25. Jasti S, Dudley WN and Goldwater E. SAS macros for testing statistical mediation in data with binary mediators or outcomes. Nurs Res 2008; 57, 118-22.

26. Cole TJ, Bellizzi MC, Flegal KM and Dietz WH. Establishing a standard definition for child overweight and obesity worldwide: international survey. BMJ 2000; 320, 12403.

27. Quah PL, Chan YH, Aris IM, et al. Prospective associations of appetitive traits at 3 and 12 months of age with body mass index and weight gain in the first 2 years of life. BMC Pediatr 2015; 15, 153.

28. de Barse LM, Tiemeier H, Leermakers ET, et al. Longitudinal association between preschool fussy eating and body composition at 6 years of age: The Generation R Study. Int J Behav Nutr Phys Act 2015; 12, 153.

29. Mallan KM, Nambiar S, Magarey AM and Daniels LA. Satiety responsiveness in toddlerhood predicts energy intake and weight status at four years of age. Appetite 2014; 74, 79-85.

30. Botton J, Heude B, Maccario J, Ducimetière P and Charles MA. Postnatal weight and height growth velocities at different ages between birth and 5 years and body composition in adolescent boys and girls. Am J Clin Nutr 2008; 87, 1760-8.

31. Dorajoo R, Ong RT, Sim X, et al. The contribution of recently identified adult BMI risk loci to paediatric obesity in a Singaporean Chinese childhood dataset. Pediatr Obes 2017; 12, e46-e50.

32. Zandoná MR, Sangalli CN, Campagnolo PdB, Vitolo MR, Almeida S and Mattevi VS. Validation of obesity susceptibility loci identified by genome-wide association studies in early childhood in South Brazilian children. Pediatric Obesity 2017; 12, 85-92. 
Figure 1. Association between obesity risk-allele score and child's eating behaviour, up to 5 years

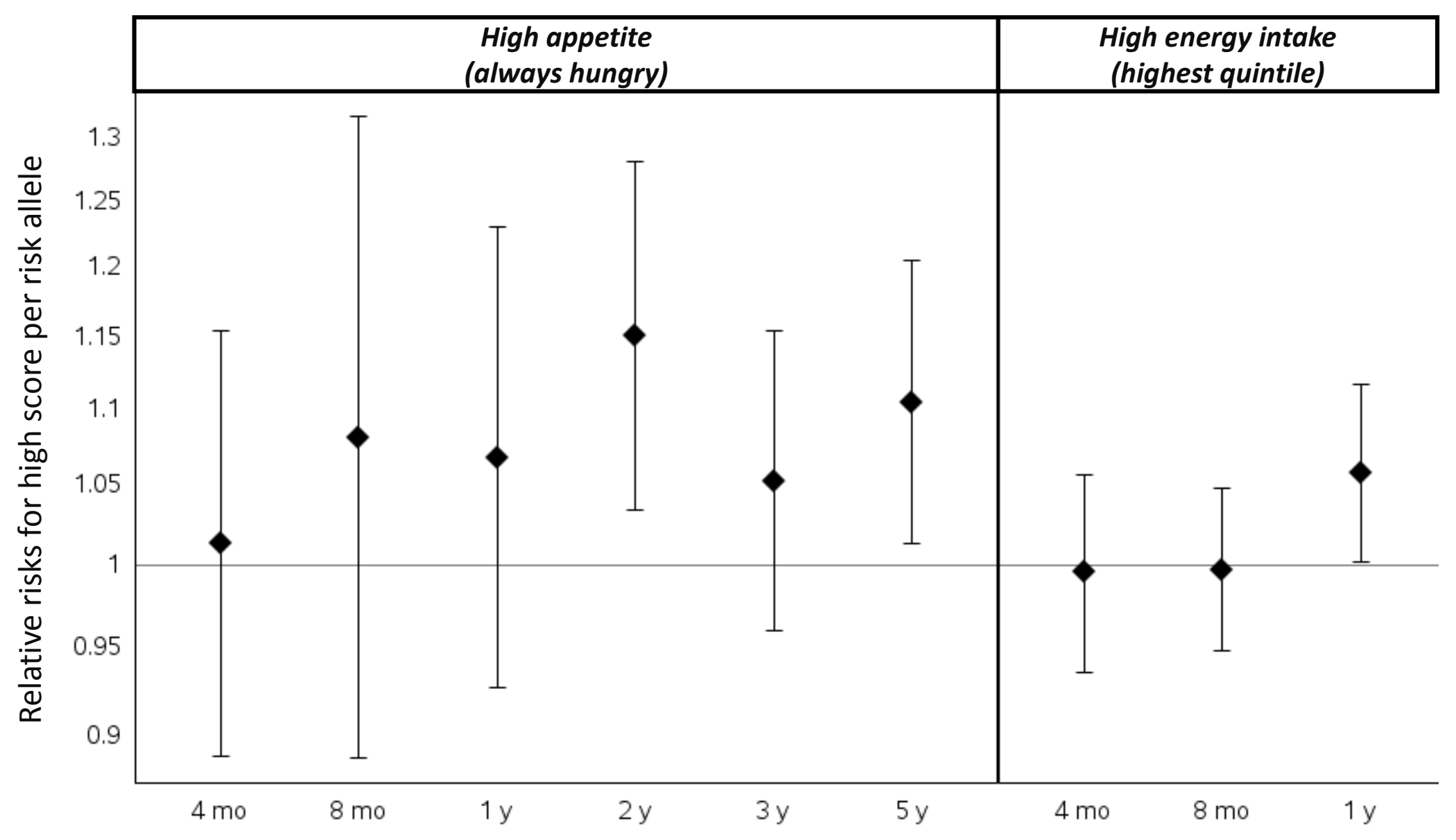


Figure legend. Results are RR [95\% CI], adjusted for recruitment's center, child's age and sex (n=1081 at 4mo, 1072 at 8 mo, 1024 at 1 year, 940 at 2 years, 892 at 3 years and 790 at 5 years). Energy intake was assessed only on infants who were not breastfed during the 3-d dietary records. (679 at $4 \mathrm{mo}, 861$ at $8 \mathrm{mo}$ and 822 at $12 \mathrm{mo}$ )

\section{Figure 2. Association between the obesity risk-allele score and child's WHO z-scores from 2 to 5 years}

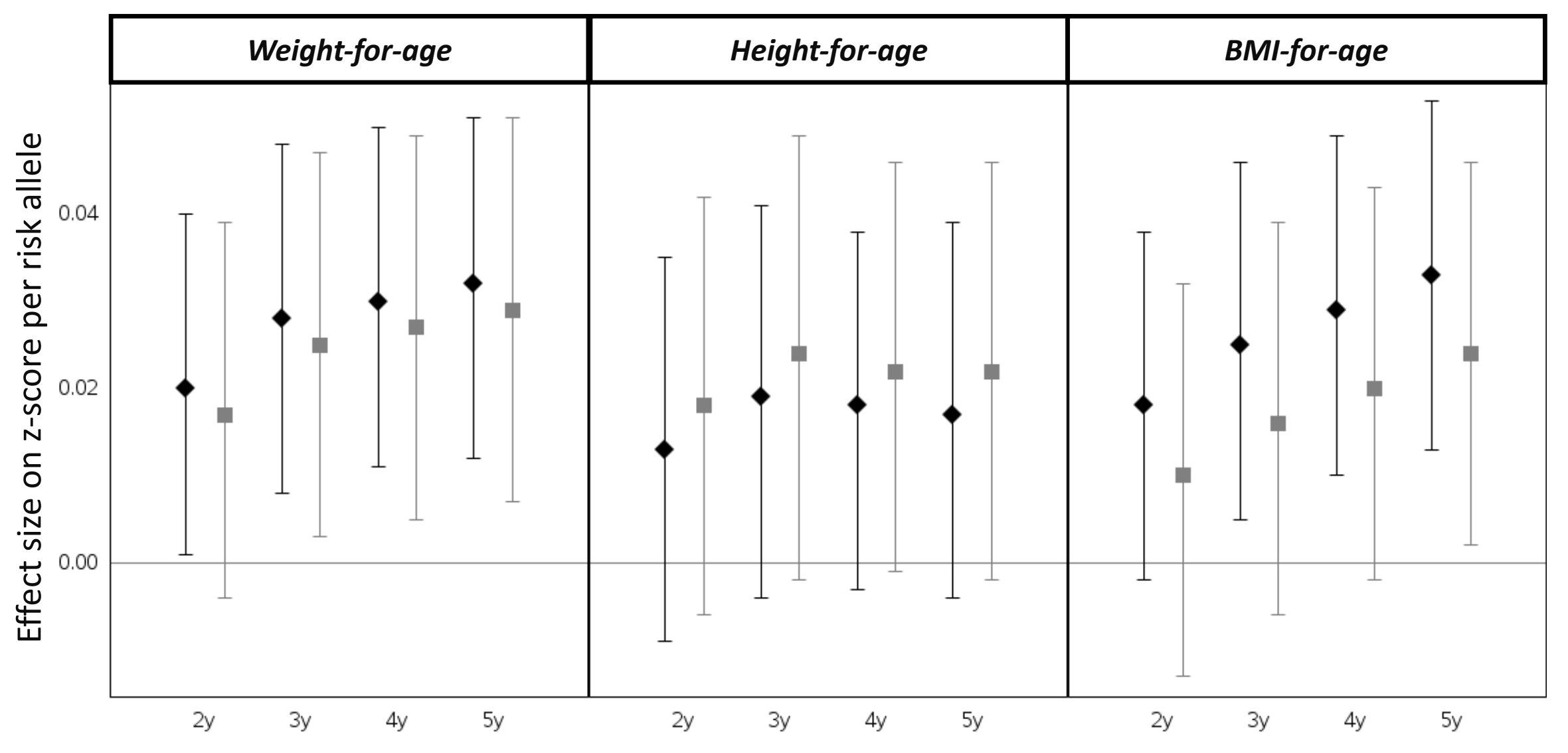


Figure legend. Results are effect size [95\% CI], adjusted for recruitment center and infant's sex (n=1142).

$\mathrm{CI}$ ], adjusted for recruitment center, infant's sex, child's age and high appetite at 2 years $(n=940)$. 
SUPPORTING INFORMATION

Table S1. BMI and eating behaviour traits of children at different assessment steps

\begin{tabular}{lllllllc} 
SNP & Locus & $\begin{array}{c}\text { Effect } \\
\text { allele }\end{array}$ & $\begin{array}{l}\text { Other } \\
\text { allele }\end{array}$ & $\begin{array}{l}\text { SNP reported } \\
\text { in GIANT }\end{array}$ & $\begin{array}{l}\text { Evidence for association with } \\
\text { childhood BMI }\end{array}$ & $\begin{array}{c}\text { LD with } \\
\text { GIANT } \\
\text { SNP (r2) }\end{array}$ & $\begin{array}{c}\text { EAF in } \\
\text { EDEN }\end{array}$ \\
\hline rs10146997 & NRXN3 & G & A & rs10150332 & Reported in Speliotes et al. & 1.0 & 0.18 \\
rs13107325 & SLC39A8 & T & C & rs13107325 & Reported in Elks et al. & - & 0.08 \\
rs1514175 & TNNI3K & A & G & rs1514175 & Reported in Speliotes et al. & - & 0.38 \\
rs1555543 & PTBP2 & C & A & rs1555543 & Reported in Speliotes et al. & - & 0.55 \\
rs17782313 & MC4R & C & T & rs571312 & Reported in den Hoed et al. & 0.96 & 0.21 \\
rs2112347 & FLJ35779 & T & G & rs2112347 & Reported in Elks et al. & - & 0.61 \\
rs2568958 & NEGR1 & A & G & rs2815752 & Reported in den Hoed et al. & 0.96 & 0.57 \\
rs4836133 & ZNF608 & A & C/G & rs4836133 & Reported in Elks et al. & - & 0.40 \\
rs4929949 & RPL27A & C & T & rs4929949 & Reported in Speliotes et al. & - & 0.45 \\
rs6548238 & TMEM18 & C & T & rs2867125 & Reported in den Hoed et al. & 1.0 & 0.81 \\
rs713586 & RBJ/POMC & C & T & rs713586 & Reported in Speliotes et al. & - & 0.44 \\
rs7640855 & CADM2 & A & G & rs13078807 & Reported in Speliotes et al. & 1.0 & 0.17 \\
rs7647305 & TRA2B & C & T & rs9816226 & Reported in den Hoed et al. & 0.72 & 0.71 \\
rs925946 & BDNF & T & G & rs10767664 & Reported in den Hoed et al. & 1.0 & 0.25 \\
rs987237 & TFAP2B & G & A & rs987237 & Reported in Elks et al. & - & 0.15 \\
rs9941349 & FTO & T & C & rs1558902 & Reported in den Hoed et al. & 0.84 & 0.38 \\
\hline
\end{tabular}

Effect allele refers to the allele associated with higher BMI in the Genetic Investigation of Anthropometric Traits (GIANT) consortium (Speliotes et al., Ref. 2).

EAF: Effect allele frequency.

LD: Linkage Disequilibrium; calculated based on 1000 Genomes pilot 1 data using SNAP (https://www.broadinstitute.org/mpg/snap/).

\section{References}

Speliotes EK, Willer CJ, Berndt SI, et al. Association analyses of 249,796 individuals reveal 18 new loci associated with body mass index. Nature Genetics. 2010;42(11):937-948.

den Hoed M, Ekelund U, Brage S, et al. Genetic susceptibility to obesity and related traits in childhood and adolescence; influence of loci identified by genome-wide association studies. Diabetes. 2010 2010;59(11):2980-2988.

Elks CE, Heude B, de Zegher F, et al. Associations between genetic obesity susceptibility and early postnatal fat and lean mass: an individual participant meta-analysis. JAMA Pediatr $2014 ; 168,1122-30$. 
$\underline{\text { Table S2. BMI and eating behaviour traits of children at different assessment steps }}$

\begin{tabular}{|c|c|c|c|c|c|c|}
\hline & 4 months & 8 months & 1 year & 2 years & 3 years & 5 years \\
\hline $\mathrm{N}$ & 1081 & 1072 & 1024 & 940 & 892 & 790 \\
\hline Age (months) & $4.3(1.0)$ & $8.3(0.8)$ & $12.1(0.7)$ & $24.4(1.3)$ & $37.9(0.9)$ & $67.7(1.9)$ \\
\hline Predicted BMI $\left(\mathrm{kg} / \mathrm{m}^{2}\right)$ & $16.9(1.3)$ & $17.1(1.3)$ & $16.8(1.2)$ & $16.2(1.2)$ & $15.9(1.2)$ & $15.4(1.3)$ \\
\hline High appetite (always hungry) $^{\mathrm{a}}$ & $3.7 \%(40)$ & $1.9 \%(20)$ & $3.1 \%(32)$ & $5.1 \%(48)$ & $7.2 \%(64)$ & $9.0 \%(71)$ \\
\hline \multicolumn{7}{|l|}{ Energy intake (kcal) } \\
\hline among children not receiving & $\mathrm{n}=680$ & $\mathrm{n}=861$ & $\mathrm{n}=822$ & & & \\
\hline breastmilk during the 3 -d record & $589(104)$ & $716(135)$ & $809(135)$ & & & \\
\hline
\end{tabular}

Values are means (sd) or \% (n).

${ }^{\text {a }}$ at 5 years, high appetite was defined as a Low Appetite Score below the $10^{\text {th }}$ percentile. 
Table S3. Association between eating behaviour and child's WHO z-scores after further adjustment on parental height, maternal smoking during pregnancy and breastfeeding duration

Outcome: Weight-for-age z-score

High energy intake (highest quintile)

$$
\begin{aligned}
& 4 \text { months } \\
& 8 \text { months } \\
& 12 \text { months }
\end{aligned}
$$

High appetite (always hungry)

$$
\begin{aligned}
& 4 \text { months } \\
& 8 \text { months } \\
& 1 \text { year } \\
& 2 \text { years } \\
& 3 \text { years } \\
& 5 \text { years }
\end{aligned}
$$

Outcome : Length/Height-for-age z-score

High energy intake (highest quintile)

$$
\begin{aligned}
& 4 \text { months } \\
& 8 \text { months } \\
& 12 \text { months }
\end{aligned}
$$

High appetite (always hungry)
4 months
8 months
1 year
2 years
3 years

$7670.04[-0.10 ; 0.19]$

$9930.32[0.04 ; 0.59]$

$9870.28[-0.10 ; 0.66]$

$9450.21[-0.10 ; 0.51]$

868

826

738

$7910.21[0.06 ; 0.35]$

$767 \quad 0.11[-0.05 ; 0.26]$

$0.13[-0.04 ; 0.29]$

$0.20[0.05 ; 0.34]$

$0.11[-0.06 ; 0.28]$

$0.18[0.03 ; 0.33]$

$0.09[-0.05 ; 0.24]$

$0.10[-0.06 ; 0.25]$

$0.10[-0.06 ; 0.26]$

$0.13[0.00 ; 0.27]$

$0.09[-0.06 ; 0.23]$

$9930.07[-0.23 ; 0.37] \quad 0.03[-0.26 ; 0.32] \quad 0.04[-0.26 ; 0.34] \quad 0.05[-0.22 ; 0.33]$

$9870.15[-0.25 ; 0.55] \quad 0.11[-0.28 ; 0.50] \quad 0.08[-0.33 ; 0.48] \quad 0.05[-0.33 ; 0.42]$

$945-0.27[-0.59 ; 0.06]$

868

826

$0.21[0.05 ; 0.36]$

$0.19[0.05 ; 0.33]$

$0.09[-0.05 ; 0.23]$

$0.09[-0.18 ; 0.37]$

$0.11[-0.26 ; 0.49]$

$0.16[0.14 ; 0.47]$

$0.24[0.02 ; 0.46]$

$-0.20[-0.45 ; 0.05]-0.20[-0.46 ; 0.07]-0.17[-0.41 ; 0.07]$

$0.11[-0.12 ; 0.34] \quad 0.09[-0.13 ; 0.30]$
$0.18[0.02 ; 0.33]$

$0.15[0.02 ; 0.29]$

$0.09[-0.05 ; 0.23]$

$0.06[-0.21 ; 0.34]$

$0.08[-0.3 ; 0.45]$

$0.14[-0.17 ; 0.44]$

$0.20[-0.04 ; 0.44]$

$0.21[-0.01 ; 0.43]$

$0.41[0.20 ; 0.61]$

$0.11[-0.05 ; 0.27]$

$0.11[-0.03 ; 0.25]$

$0.08[-0.06 ; 0.23]$

$0.07[-0.21 ; 0.35]$ $0.02[-0.36 ; 0.40]$

$-0.07[-0.38 ; 0.23]$

$-0.16[-0.41 ; 0.08]$

0.07 [-0.15;0.29] 
5 years

Outcome: BMI-for-age z-score

High energy intake (highest quintile)

$$
\begin{aligned}
& 4 \text { months } \\
& 8 \text { months } \\
& 12 \text { months }
\end{aligned}
$$

High appetite (always hungry)

4 months
8 months
1 year
2 years
3 years
5 years

993

$6290.35[0.18 ; 0.52]$

$7910.29[0.14 ; 0.44]$

$767-0.02[-0.18 ; 0.13]$

$0.03[-0.13 ; 0.18]$

$0.33[0.16 ; 0.51]$

$0.28[0.11 ; 0.45]$

$0.21[0.05 ; 0.36]$

$0.05[-0.11 ; 0.20]$

$0.21[0.04 ; 0.38]$

$0.16[0.02 ; 0.31]$

$0.06[-0.10 ; 0.21]$

$\begin{array}{llllll}993 & \mathbf{0 . 3 7}[\mathbf{0 . 0 8} ; \mathbf{0 . 6 6}] & 0.27[-0.03 ; 0.57] & 0.17[-0.13 ; 0.47] & 0.09[-0.20 ; 0.38] & 0.03[-0.26 ; 0.33] \\ 987 & 0.27[-0.13 ; 0.67] & 0.25[-0.16 ; 0.66] & 0.19[-0.22 ; 0.60] & 0.14[-0.26 ; 0.54] & 0.11[-0.29 ; 0.51] \\ 945 & \mathbf{0 . 4 9}[\mathbf{0 . 1 7} ; \mathbf{0 . 8 1}] & \mathbf{0 . 5 2}[\mathbf{0 . 2 0} ; \mathbf{0 . 8 5}] & \mathbf{0 . 4 5}[\mathbf{0 . 1 2} ; \mathbf{0 . 7 8}] & \mathbf{0 . 3 5}[\mathbf{0 . 0 2} ; \mathbf{0 . 6 7}] & 0.27[-0.05 ; 0.59] \\ 868 & & \mathbf{0 . 6 4}[\mathbf{0 . 3 8} ; \mathbf{0 . 9 0}] & \mathbf{0 . 6 0}[\mathbf{0 . 3 4} ; \mathbf{0 . 8 6}] & \mathbf{0 . 5 2}[\mathbf{0 . 2 6} ; \mathbf{0 . 7 8}] & \mathbf{0 . 4 6}[\mathbf{0 . 2 0} ; \mathbf{0 . 7 2}] \\ 826 & & & \mathbf{0 . 3 2}[\mathbf{0 . 0 8} ; \mathbf{0 . 5 6}] & \mathbf{0 . 2 9}[\mathbf{0 . 0 5} ; \mathbf{0 . 5 2}] & \mathbf{0 . 2 7}[\mathbf{0 . 0 3} ; \mathbf{0 . 5 0}] \\ 738 & & & & & \mathbf{0 . 3 5}[\mathbf{0 . 1 3} ; \mathbf{0 . 5 7}]\end{array}$

$0.16[-0.01 ; 0.33]$

$0.14[-0.01 ; 0.28]$

$0.06[-0.09 ; 0.21]$

$0.30[0.09 ; 0.50]$

$0.35[0.13 ; 0.57]$

Results are effect size [95\% CI] of high level of the considered appetitive trait on WHO z-score, adjusted for recruitment centre, infant's sex, age at eating behaviour assessment, parental height, maternal smoking during pregnancy and breastfeeding duration. Significant results are highlighted in bold.

${ }^{a}$ at 5 years, high appetite was defined as a Low Appetite Score below the $10^{\text {th }}$ percentile. 
Figure S1. Figure depicting the mediation analyses.

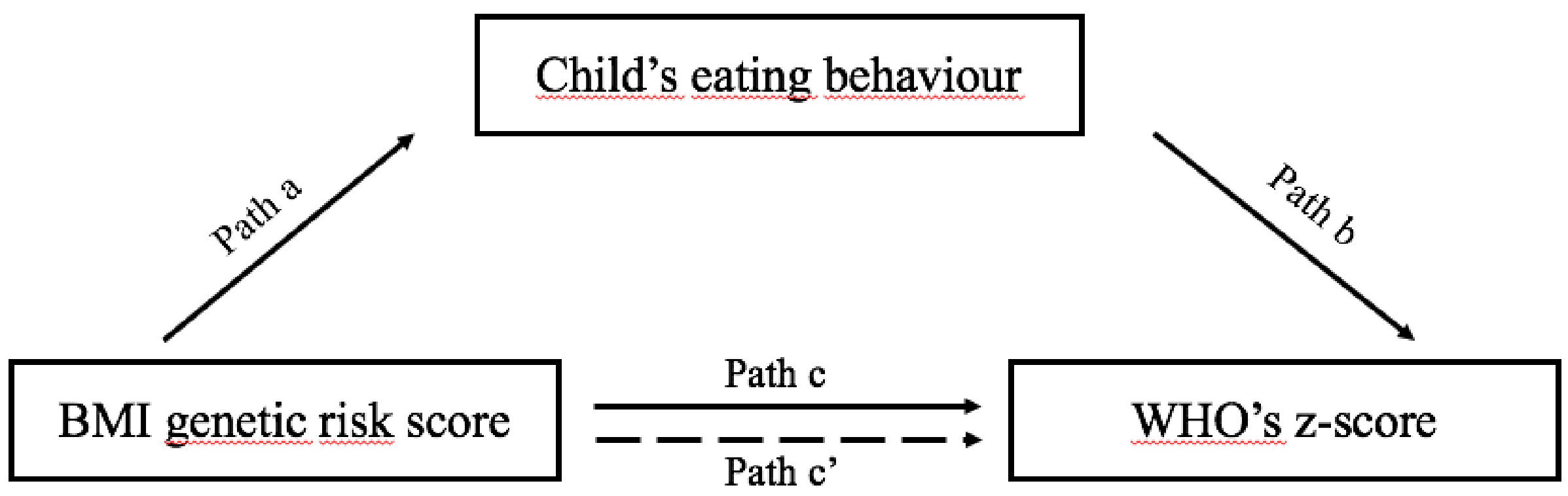

Path a shows the association between the BMI genetic risk score and eating behaviour

Path b shows the association between eating behaviour and WHO's z-score

Path c shows the relationship between the BMI genetic risk score and WHO's z-score

Path c' shows the relationship between the BMI genetic risk score and WHO's z-score, adjusted

for the considered eating behaviour 